\title{
Comparative Assessment of the Diagnostic Performance of PCA3 in Urinary Sediments and Exosomes for Prostate Cancer in Chinese Population with the Total PSA range of 4-10 $\mathrm{ng} / \mathrm{ml}$.
}

\author{
Zhenqiang Fang \\ Third Military Medical University Second Affiliated Hospital: Xinqiao Hospital \\ Fan He \\ Third Military Medical University Second Affiliated Hospital: Xinqiao Hospital \\ Huan Feng \\ Third Military Medical University Second Affiliated Hospital: Xinqiao Hospital \\ Weishen Jia \\ Third Military Medical University Second Affiliated Hospital: Xinqiao Hospital \\ Mengjia Sun \\ Third Military Medical University: Army Medical University \\ Tingting Chen \\ Third Military Medical University Second Affiliated Hospital: Xinqiao Hospital \\ Ronghua Wu \\ Third Military Medical University Second Affiliated Hospital: Xinqiao Hospital \\ Weihua Fu ( $\square$ fuweihua80@tmmu.edu.cn )
}

Third Military Medical University Second Affiliated Hospital: Xinqiao Hospital https://orcid.org/0000-0003-1846-1959

Research article

Keywords: prostate cancer antigen 3, prostate specific antigen, prostate cancer, Chinese population, diagnosis, grey zone

Posted Date: December 4th, 2020

DOI: https://doi.org/10.21203/rs.3.rs-120215/v1

License: @ (i) This work is licensed under a Creative Commons Attribution 4.0 International License. Read Full License 


\section{Abstract}

Background: The diagnostic value of prostate cancer antigen-3 (PCA3) in urine sediment and exosome in Chinese patients with total prostate specific antigen (PSA) ranging from 4-10ng/ml.

Methods: Serum and urine samples were collected from consecutive eligible patients. The PCA3 and PSA mRNA were tested by quantitative real-time PCR.

Results: Overall 130 patients were involved in this study. 113 cases in urinary sediments group and 103 cases in urinary exosomes group were finally analyzed. 24 of 130 patients (18.5\%), 20 of 113 patients (17.7\%) and 17 of 103 patients (16.5\%) were diagnosed as PCa in the three groups, respectively. The PCA3 score and PSAD of patients with positive biopsy results were significantly higher than those with negative biopsy results in both the urinary sediments and exosomes groups, but no differences between two urinary substrates groups. The ROC analysis showed the higher values of AUC of the PCA3 score in urinary sediments and exosomes than that of serum PSA ( 0.728 vs $0.540, P=0.0402 ; 0.740$ vs $0.540, P=0.0263)$, but no significant difference in term of $A U C$ of PCA3 between two urinary substrates groups ( 0.728 vs $0.740, P=0.9000)$, as well as when compared the AUC of the PCA3 score with that of \% fPSA and PSAD $(P>0.05)$.

Conclusion: The current evidence suggests that diagnostic performances of PCA3 in urinary sediments and exosomes were not significant different, but both were superior to serum PSA in Chinese patients with PSA 4-10ng/ml.

\section{Background}

Prostate cancer $(\mathrm{PCa})$ is the most common male malignant cancer in western countries ${ }^{[1]}$. Because of racial and genetic differences, the incidence of $\mathrm{PCa}$ is much lower in Asian countries, but the incidence is rapidly increasing in recent years, especially in developing Asian countries such as in China ${ }^{[2]}$. According to the Chinese cancer statistics, PCa is the fastest increasing malignant in terms of incidences between 2000 and 2011 , ranks as the sixth most prevalent male cancer after lung, stomach, colorectum, liver and esophagus cancer ${ }^{[3]}$. The cause of the increasing trend is multifactorial. One of the crucial factors is the gradual implementation of population-based cancer screening, which significantly improves the detection rate of PCa ${ }^{[3]}$.

So far, the PCa screening still relies primarily on serum prostate-specific antigen (PSA)-based test, which indicates prostate biopsy for histopathological diagnosis. However, PSA elevation is not peculiar for PCa, serum PSA level also rises when the development of benign prostatic hyperplasia (BPH) and prostatitis. The low PCa-specificity of PSA leads to a high rate of false-positive biopsies up to $60.6 \%-75.8 \%$ [4] . Furthermore, serum PSA seems not to ensure an absolutely safe cut-off value. Thompson IM, et al. ${ }^{[5]}$ found that about $15 \%$ of the men with a PSA $\leq 4.0 \mathrm{ng} / \mathrm{mL}$ have PCa through prostate biopsy. Because of its limitations, PSA-based screening for PCa has remained controversial. The development of more efficient biomarkers for PCa is urgently needed.

Noninvasive urine-based biomarkers for PCa screening attracted much attention in recent years. Among which, prostate cancer antigen 3 (PCA3) has been extensively evaluated and recognized as one of the most promising candidates. The accumulating evidence suggested that PCA3 showed better performance than serum PSA in terms of specificity and biopsy outcome prediction ${ }^{[6]}$. In addition to the whole urine or urinary sediments, urine exosomes is recognized as a novel substrate to evaluate the diagnostic value of PCA3 for PCa. Compared with urinary sediments, exosomes seemed to contain more sources of biomarkers ${ }^{[7]}$. However, few groups have investigated the role of urine-based PCA3 in Chinese population. Recent research suggested that the diagnostic accuracy of PCA3 in Chinese patients seem to be lower than that in western populations ${ }^{[8]}$. The clinical value of PCA3 in the PCa diagnosis should be further evaluated in Chinese population.

In this study, 130 patients with the serum PSA levels in "gray zone" (4-10 ng/mL) were involved, who underwent initial prostate biopsies at XinQiao Hospital. The levels of PCA3 mRNA were measured in urinary sediments and exosomes after digital rectal examination (DRE) with the quantitative real-time RT-PCR. The effectiveness of PCA3 in biopsy outcome prediction was assessed, and compared with the PSA-based parameters in two types of substrates.

\section{Methods Study Design}

All participants were consecutively recruited from XinQiao Hospital between May 2016 and June 2019. The inclusion criteria are as follows: (1) older than 50 years old, (2) the serum PSA of $4-10 \mathrm{ng} / \mathrm{ml}$, (3) receiving initial prostate biopsy, (4) understanding the purpose and risks of the study and volunteering for participation in the study. Participants were excluded if they had urinary tract infection, recent instrumentation or catheterization of the urethra, other known malignant cancers, and those receiving medical therapy known to affect serum PSA levels within 6 months, such as finasteride or hormonal treatment. This protocol was approved by the Medical Ethics Committee of XinQiao Hospital (No. AF/SC-08/1.0). All participants signed an informed consent form before enrollment.

Peripheral blood samples were obtained prior to DRE and prostate biopsies. DRE was performed by the same urologist with a standardized protocol as described by Groskopf J. et al: stable pressure to depress the prostate surface approximately $1 \mathrm{~cm}$ from base to apex and from lateral to median line, three times for each lobe ${ }^{[9]}$. After DRE, first catch urine were collected and divided into two samples for urinary sediments and exosomes substrate to test PSA and PCA3 mRNA. The three dimensions of prostate were measured by transrectal ultrasonography (TRUS) at the beginning of 14-cores systematic prostate biopsies. Prostate volume (PV) was calculated using the formula: $(\pi / 6) \times$ anterior-posterior diameter $\times$ transverse diameter $\times$ superior-inferior diameter. PSA density (PSAD) and percent free PSA (\%fPSA) were evaluated. Pathology results of prostate biopsy were proclaimed at the end of this study. 


\section{Urine Sample Preparation}

Urinary sediment and exosomes were separated from urine samples within 2 hours after collection. Briefly, urine samples in the $50 \mathrm{~mL}$ transfer tube containing EDTA were centrifuged at $1,000 \times \mathrm{g}$ for $10 \mathrm{~min}$ at $4{ }^{\circ} \mathrm{C}$ to remove whole cells and large aggregates, followed by $18,000 \times \mathrm{g}$ for $10 \mathrm{~min}$ at $4{ }^{\circ} \mathrm{C}$ to discard additional cellular debris, organelles. The acquired urinary sediments were washed twice with ice-cold Phosphate buffered saline (PBS), then snap-frozen in liquid nitrogen and stored at $-80^{\circ} \mathrm{C}$ for further use. Additionally, approximate $50 \mathrm{~mL}$ exosomes-containing supernatant were ultracentrifuged sequentially at $200,000 \times \mathrm{g}$ for 1 hour at $4^{\circ} \mathrm{C}$, filtrated with a $0.8 \mu \mathrm{m}$ pore Supor syringe filter device (Millipore, Bedford, MA, USA). The acquired exosome-containing pellet was washed twice with ice-cold PBS, and re-pelleted at $100,000 \times \mathrm{g}$ for 1 hour at $4^{\circ} \mathrm{C}$, then re-suspended in PBS for maximal retrieval, and finally subjected to DNase to remove extraneous nucleic acids. Electron microscopy and Immunoblotting analysis were performed to identify exosomes.

\section{Quantitative Real-time Pcr (qrt-pcr)}

Total RNA was isolated from urinary sediments and exosome samples using RNAisoPlus (Takara, \#9108), according to the manufacturer's instructions. Reverse transcription of the extracted RNA to corresponding complementary DNA was performed using PrimeScript RT reagent Kit with gDNA Eraser (Takara Bio, Inc., Otsu, Japan). RT-qPCR was performed with QuantiNovaTM SYBR® Green PCR Kit (Qiagen GmbH, Hilden, Germany) on an Applied Biosystems 7900HT Real-Time PCR System. The housekeeping gene, GAPDH, was used as loading controls. The following forward (F) primers, and reverse (R) primers were used: PSA forward-AGCATTGAACCAGAGGAGTTCT, reverse-CCCGAGCAGGTGCTTTTG; PCA3 forward-CCCAACGCATGTCTGAGATCC, reverseGTCCAAAGCAACA GAGCAGAGAG. The results were analyzed by relative quantitative method, using PSA mRNA as an internal reference. The cut-off value for an adequate amount of PSA mRNA was set at 1000 copies to ensure sufficient amount of prostate specific transcripts and better normalized PCA3 signals. No exclusion was done based on this criterion to test the analytical performance. The corrected exosome PCA3 expression levels were calculated as PCA3 mRNA/PSA mRNA $\times 1000=2^{\mathrm{Ct}(\mathrm{PSA})-\mathrm{Ct}(\mathrm{PCA} 3)} \times 1000$. Each experiment was conducted in triplicate.

\section{Statistical analysis}

All data were shown as the average \pm standard deviation (Mean \pm SD) or median (interquartile range) and each experiment was repeated at least three times independently. All Statistical analyses were performed using the SPSS 18.0 and MedCalc 19.0. The statistical significance of differences between groups was examined by student's t test or Mann Whitney U-test. The data were summarized in a receiver operating characteristic (ROC) curve. The diagnostic accuracy of each biomarker was quantified using the area under the curve (AUC) and 95\% confidence intervals (Cls) were computed. Based on which, the optimal cut-off value was determined where the Younden index (calculated as sensitivity + specificity-1) was maximum. The significant difference between the AUCs of each pair of parameters (PSA, \% fPSA, PSAD and PCA3) was calculated by the Z-test. Two-sided test was used. A p-value of less than 0.05 was considered to be statistically significant.

\section{Results}

\section{Patient characteristics}

The flow diagram of this study was shown in Fig. 1. A total of 130 patients were involved in this study. Because of insufficient RNA, 17 samples in the urinary sediments group and 27 samples in the urinary exosomes group were excluded. Finally, a total of 113 patients and 103 patients were analyzed in the urinary sediments group and the urinary exosomes group, respectively. The clinical characteristics of each group were summarized in Table 1 . According to the biopsy outcomes, 24 of all 130 patients (18.5\%) were diagnosed as PCa, 20 of 113 patients (17.7\%) in the urinary sediments group and 17 of 103 patients (16.5\%) in the urinary exosomes group were positive biopsy outcomes. The significant differences in age and serum PSA were not founded between PCa groups and non-PCa groups, as well as between two urinary substrates groups $\left(P^{*}>0.05, P^{\#}>0.05\right.$, respectively).

Table 1

Clinical characteristics of patients

\begin{tabular}{|c|c|c|c|c|c|c|c|c|c|}
\hline \multirow[t]{2}{*}{ Median(IQR) } & \multicolumn{3}{|l|}{ Serum PSA } & \multicolumn{3}{|c|}{ Urinary sediments } & \multicolumn{3}{|c|}{ Urinary exosomes } \\
\hline & Positive & Negative & $P^{*}$ & Positive & Negative & $P^{*}$ & Positive & Negative & F \\
\hline Number & 24 & 106 & & 20 & 93 & & 17 & 86 & \\
\hline Age(years)Mean(s.d.) & $66.25(9.94)$ & 64.12(10.35) & 0.362 & $66.90(10.96)$ & 63.62(10.45) & 0.208 & $66.47(11.37)$ & $63.22(10.52)$ & 2 \\
\hline PSA (ng/ml) & $7.03(6.19,7.91)$ & $6.90(5.28,8.18)$ & 0.541 & $7.03(6.19,7.92)$ & $6.93(5.27,8.18)$ & 0.600 & $6.95(5.87,7.71)$ & $6.90(5.28,8.18)$ & C \\
\hline$\% f P S A(\%)$ & $14.7(10.0,21.9)$ & $13.6(9.2,17.4)$ & 0.368 & $13.8(9.0,21.9)$ & $13.6(9.1,16.5)$ & 0.452 & $15.0(9.7,22.2)$ & $13.6(9.6,16.5)$ & $c$ \\
\hline $\operatorname{PSAD}\left(\mathrm{ng} / \mathrm{ml}^{2}\right)$ & $0.25(0.16,0.31)$ & $0.16(0.11,021)$ & 0.006 & $0.23(0.16,0.30)$ & $0.16(0.11,0.21)$ & 0.012 & $0.23(0.11,0.30)$ & $0.15(0.11,0.20)$ & $c$ \\
\hline РСАЗ & & & & 157(99.5,238.5) & $83(42.5,148)$ & 0.001 & $155(87,230.8)$ & $74(36,143)$ & C \\
\hline
\end{tabular}

IQR: interquartile range; s.d.: standard deviation; PSA: prostate-specific antigen; \%fPSA: percent free prostate-specific antigen; PSAD: prostate-specific antigen cancer antigen 3; $\mathrm{P}^{*}$ : comparing between $\mathrm{PCa}$ and non-PCa patients; $\mathrm{P}^{\#}$ : comparing between two urinary substrates groups 
In the total 130 patients, there were not significant differences in serum PSA and \%fPSA between the PCa group and the non-PCa group $(p=0.541$, $p=0.362$, respectively). However, the median PSAD was higher in the PCa group compared to the non-PCa group $(0.25 v s 0.16, P=0.006)($ Table 1$)$. Similar results were found in two urinary substrates groups $(P=0.012, p=0.042$, respectively). The diagnostic effectiveness of serum PSA-based biomarkers was evaluated by the ROC analysis (Fig. 2a). The AUCs of PSA, \%fPSA and PSAD for predicting PCa were 0.540 (95\%Cl: $0.451-0.628), 0.559$ (95\%Cl: 0.469-0.646), and 0.681(95\% Cl: 0.592-0.759), respectively, there were not significant differences in AUC among the three PSA-based biomarkers (Table 2). The statistical optimal cut-off of serum PSA is $6.05 \mathrm{ng} / \mathrm{ml}$; the corresponding sensitivity and specificity were $79.17 \%$ and $33.96 \%$, respectively. Sensitivity and specificity of \%fPSA were $41.67 \% 79.25 \%$ at the optimal cut-off value of 0.180 , and at an optimal cut-off value of 0.222 , sensitivity and specificity of PSAD were $58.33 \%$ and $80.19 \%$, respectively.

Table 2

Diagnostic performance of serum PSA-based biomarkers and urinary PCA3

\begin{tabular}{|c|c|c|c|c|c|}
\hline & Number & AUC(95\%Cl) & Cut off & Sensitivity & Specificity \\
\hline PSA & 130 & $0.540(0.451,0.628)$ & 6.05 & 79.17 & 33.96 \\
\hline$\% f P S A$ & 130 & $0.559(0.469,0.646)$ & 18.0 & 41.67 & 79.25 \\
\hline PSAD & 130 & $0.681(0.592,0.759)$ & 0.22 & 58.33 & 80.19 \\
\hline PCA3 in sediments & 113 & $0.728(0.636,0.807)$ & 83 & 85.00 & 50.54 \\
\hline PCA3 in exosomes & 103 & $0.740(0.644,0.821)$ & 79 & 82.35 & 53.49 \\
\hline \multirow[t]{10}{*}{$\mathrm{P}$} & \multicolumn{3}{|c|}{ PSA vs \%fPSA } & \multicolumn{2}{|l|}{$P=0.8418$} \\
\hline & \multicolumn{3}{|c|}{ PSA vs PSAD } & \multicolumn{2}{|l|}{$P=0.1251$} \\
\hline & \multicolumn{3}{|c|}{$\%$ fPSA vs PSAD } & \multicolumn{2}{|l|}{$P=0.2059$} \\
\hline & \multicolumn{3}{|c|}{ PCA3 in sediments vs PCA3 in exosomes } & \multicolumn{2}{|l|}{$P=0.9000$} \\
\hline & \multicolumn{3}{|c|}{ PCA3 in sediments vs PSA } & \multicolumn{2}{|l|}{$P=0.0402$} \\
\hline & \multicolumn{3}{|c|}{ PCA3 in sediments vs \%fPSA } & \multicolumn{2}{|l|}{$P=0.0786$} \\
\hline & \multicolumn{3}{|c|}{ PCA3 in sediments vs PSAD } & \multicolumn{2}{|l|}{$P=0.6121$} \\
\hline & \multicolumn{3}{|c|}{ PCA3 in exosomes vs PSA } & \multicolumn{2}{|l|}{$P=0.0263$} \\
\hline & \multicolumn{3}{|c|}{ PCA3 in exosomes vs \% fPSA } & \multicolumn{2}{|l|}{$P=0.0558$} \\
\hline & \multicolumn{3}{|c|}{ PCA3 in exosomes vs PSAD } & \multicolumn{2}{|l|}{$P=0.5208$} \\
\hline
\end{tabular}

The significant difference was calculated by the Z-test; AUC: area under the ROC curve; PSA: prostate-specific antigen; \%fPSA: percent free prostatespecific antigen; PSAD: prostate-specific antigen density; PCA3: prostate cancer antigen 3.

\section{Diagnostic Performance Of Pca3 In Urinary Sediments And Exosomes}

As the results shown in Table 1, there was not significantly different in the PCA3 score between the urinary sediments group and the urinary exosomes group $(p=0.2434)$. However, the PCA3 score were both significantly higher in men with positive biopsy outcomes than those with negative biopsy outcomes in two urine substrates groups ( 157 vs. $83 ; p=0.001$ and 155 vs. $74 ; p=0.002$, respectively). According to the results of the ROC analysis (Fig. $2 b$ ), AUC of PCA3 was $0.728(95 \% \mathrm{Cl}: 0.636-0.807)$ in urinary sediments and 0.740 (95\% Cl: $0.644-0.821)$ in urinary exosomes (Table 2 ). No significant difference in AUC was observed between two urine substrates groups $(p=0.9000)$. In the urinary sediments group, the statistical optimal cut-off of the PCA3 score was 83 ; sensitivity and specificity were $85.00 \%$ and $50.54 \%$, respectively. Similar results were found in the urinary exosomes group, the sensitivity and specificity were $82.35 \%$ and $53.49 \%$, respectively at the optimal cut-off value of 79 (Table 2 ).

Compared with serum PSA-based biomarkers, the AUC of PCA3 score was only significantly higher than that of serum PSA in the urinary sediments and exosomes groups ( $p=0.0402$ and $p=0.0263$, respectively), but there were no significant differences when comparing with the AUC of \% fPSA and PSAD (both $P>0.05)$ (Table 2).

\section{Discussion}

The primary drawback of serum PSA as a biomarker of PCa screening is the low specificity, especially in the PSA "gray area" (4.0-10.0 ng/mL). Compared with Western populations, its specificity for PCa seemed to be overall lower in Chinese populations. About $25 \%$ of patients with PSA in gray area were diagnosed as PCa, but approximately $40 \%$ in US clinical practice ${ }^{[10]}$. The data from a collaborative report of the Asian population agreed with the viewpoint, which revealed that the detection rate of PCa is general below $25 \%$ in Asian men with serum PSA $4-10 \mathrm{ng} / \mathrm{mL}$, even below $10 \%$ in Indonesia men ${ }^{[11]}$. In this study, about $18.5 \%$ cases were positive biopsy outcomes. In addition, our results suggested that the diagnostic performance of PSAD seems to be better than PSA, The AUCs of PSA and PSAD for predicting PCa were 0.540 (95\%Cl: $0.451-0.628)$ and 0.680 (95\%Cl: $0.592-0.759)$, respectively. A recent study showed 
similar results that the AUC for predicting PCa was 0.55 for PSA and 0.69 for PSAD in 2,162 men with serum PSA 4-10 ng/mL, of whom $56 \%$ were African American ${ }^{[12]}$. The significant difference in AUC was found in this report, but it was not shown in the present study.

The diagnostic value of urine PCA3 gene-based analysis for PCa has been extensively investigated in western populations. In this study, the diagnosis performances of PCA3 were evaluated in the urinary sediments from 113 Chinese patients with serum PSA 4-10 ng/ml. The PCA3 showed an AUC of 0.728 in the urinary sediments, which is slightly lower than that reported in two previous studies, where the AUC values of PCA3 were 0.734 and 0.750 in Chinese males with PSA $4-10 \mathrm{ng} / \mathrm{ml}^{[8,13]}$. It should be noted that these three studies all suggested that the diagnostic accuracy of PCA3 was superior to that of PSA. The AUC values of PCA3 remain inconsistent among some studies of Western populations with PSA $4-10 \mathrm{ng} / \mathrm{ml}$. Rigau M et al. ${ }^{[14]}$ found an AUC of only $0.61 \mathrm{for}$ PCA3 and Perdonà $S$ et al. ${ }^{[15]}$ described a high AUC of 0.873 . This may be associated with the valuation procedures of the level of PCA3 mRNA, in addition to other variables in these studies, such as ethnic, small sample sizes. The PCA3 was generally measured with the Progensa PCA3 assay In Western studies, but with quantitative real-time RT-PCR assay in Chinese studies. However, Filella et al. thought that there was no significant difference between two methods used to assess the diagnostic effectiveness of PCA3 [16].

In the present study, the diagnostic value of PCA3 in the urinary exosomes was also assessed in 103 patients with serum PSA 4-10 ng/ml. Exosomes are the internal vesicles secreted from various cell types, including cancer cells. They are 30-200 nm in diameter, and contain the proteins, mRNAs and microRNAs of the derived cells. Nilsson et al. firstly reported that PCA3 could be detected in exosomes in urine of PCa patients in 2009 [17]. Since then, few studies have investigated the diagnostic value of PCA3 in exosomes for PCa. In the study of Dijkstra et al., 24 (80\%) of 30 patients with PSA > $3 \mathrm{ng} / \mathrm{ml}$ meet the predetermined cutoff of the sufficient amount of mRNA to assess the diagnostic performance of PCA3 ${ }^{[7]}$. The rate of assessable exosomes samples was similar to the results of Hendriks et al. and the present study ${ }^{[18]}$. However, the AUC of PCA3 in urinary exosomes was 0.64 (95\% Cl $\left.0.41-0.88\right)$, that is lower than our results 0.740 (95\% Cl: $0.644-0.821)$ ). The difference of AUC values may result from the heterogenicities in two studies, such as ethnic, small sample sizes. Consequently, the diagnostic value of PCA3 in urinary exosomes should be further evaluated. It should be noted that the exciting results have been recently reported in a series of studies of ExoDx Prostate test, which was used to predict the outcomes of initial biopsy and high-grade PCa through analyzing the TMPRSS2: ERG fusion gene and PCA3 in the exosomes of first-catch urine samples without prostate manipulation [19-21]. ExoDx Prostate test has been commercially available as a urinary test for the detection of PCa in Western countries. However, the report of Chinese populations using ExoDx Prostate test has not been found.

There are several limitations in this study should be acknowledged. First, the manual quantitative real-time RT-PCR is used to test PCA3 and PSA mRNA in urine samples, instead of the commercial PROGENSA ${ }^{\circ}$ PCA3 test. Second, it is a single-center trial with limited participants. More convincing results should come from future large sample size and multicenter studies.

\section{Conclusion}

The current evidence suggests that the diagnostic performances of PCA3 in urinary sediments and exosomes were not significant different, but both were superior to serum PSA in Chinese patients with PSA 4-10 ng/ml. However, future multi-center randomized controlled trials are needed to recognize the diagnostic value of PCA3 in urinary sediments and exosomes for prostate cancer in Chinese population.

\section{Abbreviations}

$\mathrm{BPH}$

Benign prostatic hyperplasia

PCA3

Prostate cancer antigen 3

DRE

Digital rectal examination

TRUS

Transrectal ultrasound

PV

Prostate volume

PBS

Phosphate buffered saline

ROC

receiver operating characteristic

\section{Declarations}

\section{Ethics approval and consent to participate}

All procedures performed in studies involving human participants were conducted in accordance with the ethical standards of the institutional and/or national research committee and the 1964 Helsinki declaration and its later amendments or comparable ethical standards. This study protocol was approved by the Medical Ethics Committee of XinQiao Hospital (No. AF/SC-08/1.0).

\section{Consent for publication}


Not applicable.

\section{Availability of data and material}

Data will be available by contacting the corresponding author. All strains and reagents used in the studies are available upon request.

\section{Competing interests}

The authors declare that they do not have any conflict of interest

\section{Funding}

This study was funded by a Science and Technology Innovation Foundation of Chongqing Science and Technology bureau (Grant number cstc2015shmszx120018) and a Clinical Innovation Foundation of XinQiao Hospital (Grant number 2015YLC24).

\section{Author information}

Affiliations

\section{Department of Urology, XinQiao Hospital, Army Medical University, Chongqing, China.}

Zhenqiang Fang, Fan He, Huan Feng, Weishen Jia, Tingting Chen, Ronghua Wu, \& Weihua Fu

College of Basic Medical Sciences, Army Medical University, Chongqing, China. Mengjia Sun

Contributions

F He and ZQ Fang wrote the original draft. WS Jia, H Feng, TT Chen, RH Wu and MJ Sun performed data curation. WH Fu was responsible for project administration.

Corresponding author

Correspondence to Weihua Fu.

\section{Acknowledgements}

The authors would like to thank everyone who participated in the research and for taking care of the patients.

\section{References}

1. Bray F, Ferlay J, Soerjomataram I, et al. Global cancer statistics 2018: GLOBOCAN estimates of incidence and mortality worldwide for 36 cancers in 185 countries. CA Cancer J Clin. 2018;68(6):394-424.

2. Kimura T, Egawa S. Epidemiology of prostate cancer in Asian countries. Int J Urol. 2018;25(6):524-31.

3. Chen W, Zheng R, Baade PD, et al. Cancer statistics in China, 2015. CA Cancer J Clin. 2016;66(2):115-32.

4. Fenton JJ, Weyrich MS, Durbin S, et al. Prostate-Specific Antigen-Based Screening for Prostate Cancer: Evidence Report and Systematic Review for the US Preventive Services Task Force. JAMA. 2018;319(18):1914-31.

5. Thompson IM, Pauler DK, Goodman PJ, et al. Prevalence of prostate cancer among men with a prostate-specific antigen level < or $=4.0 \mathrm{ng}$ per milliliter. $\mathrm{N}$ Engl J Med. 2004;350(22):2239-46.

6. Ploussard G, de la Taille A. The role of prostate cancer antigen 3 (PCA3) in prostate cancer detection. Expert Rev Anticancer Ther. 2018;18(10):1013-20.

7. Dijkstra S, Birker IL, Smit FP, et al. Prostate cancer biomarker profiles in urinary sediments and exosomes. J Urol. 2014;191(4):1132-8.

8. Wang FB, Chen R, Ren SC, et al. Prostate cancer antigen 3 moderately improves diagnostic accuracy in Chinese patients undergoing first prostate biopsy. Asian J Androl. 2017;19(2):238-43.

9. Groskopf J, Aubin SM, Deras IL, et al. APTIMA PCA3 molecular urine test: development of a method to aid in the diagnosis of prostate cancer. Clin Chem. 2006;52(6):1089-95.

10. Chen R, Sjoberg DD, Huang Y, et al. Prostate Specific Antigen and Prostate Cancer in Chinese Men Undergoing Initial Prostate Biopsies Compared with Western Cohorts. J Urol. 2017;197(1):90-6.

11. Chen R, Ren S, Chinese Prostate Cancer Consortium. et al. Prostate cancer in Asia: A collaborative report. Asian J Urol. 2014;1(1):15-29.

12. Aminsharifi A, Howard L, Wu Y, et al. Prostate Specific Antigen Density as a Predictor of Clinically Significant Prostate Cancer When the Prostate Specific Antigen is in the Diagnostic Gray Zone: Defining the Optimum Cutoff Point Stratified by Race and Body Mass Index. J Urol. 2018;200(4):758-66.

13. Zhou Y, Li Y, Li X, et al. Urinary Biomarker Panel to Improve Accuracy in Predicting Prostate Biopsy Result in Chinese Men with PSA 4-10 ng/mL. Biomed Res Int. 2017; 2017: 2512536

14. Rigau M, Ortega I, Mir MC, et al. A three-gene panel on urine increases PSA specificity in the detection of prostate cancer. Prostate. 2011;71(16):1736-45.

15. Perdonà S, Cavadas V, Di Lorenzo G, et al. Prostate cancer detection in the "grey area" of prostate-specific antigen below $10 \mathrm{ng} / \mathrm{ml}$ : head-to-head comparison of the updated PCPT calculator and Chun's nomogram, two risk estimators incorporating prostate cancer antigen 3. Eur Urol. 2011;59(1):81- 
16. Filella X, Foj L, Milà M, et al. PCA3 in the detection and management of early prostate cancer. Tumour Biol. 2013;34(3):1337-47.

17. Nilsson J, Skog J, Nordstrand A, et al. Prostate cancer-derived urine exosomes: a novel approach to biomarkers for prostate cancer. Br $\mathrm{J}$ Cancer. 2009;100(10):1603-7.

18. Hendriks RJ, Dijkstra S, Jannink SA, et al. Comparative analysis of prostate cancer specific biomarkers PCA3 and ERG in whole urine, urinary sediments and exosomes. Clin Chem Lab Med. 2016;54(3):483-92.

19. Donovan M, Noerholm M, Bentink S, et al. A molecular signature of PCA3 and ERG exosomal RNA from non-DRE urine is predictive of initial prostate biopsy result. Prostate Cancer Prostatic Dis. 2015;18:370-5.

20. McKiernan J, Donovan MJ, O'Neill V, et al. A Novel Urine Exosome Gene Expression Assay to Predict High-grade Prostate Cancer at Initial Biopsy. JAMA Oncol. 2016;2(7):882-9.

21. McKiernan J, Donovan MJ, Margolis E, et al. A Prospective Adaptive Utility Trial to Validate Performance of a Novel Urine Exosome Gene Expression Assay to Predict High-grade Prostate Cancer in Patients with Prostate-specific Antigen 2-10 ng/ml at Initial Biopsy. Eur Urol. 2018;74(6):731-8.

\section{Figures}

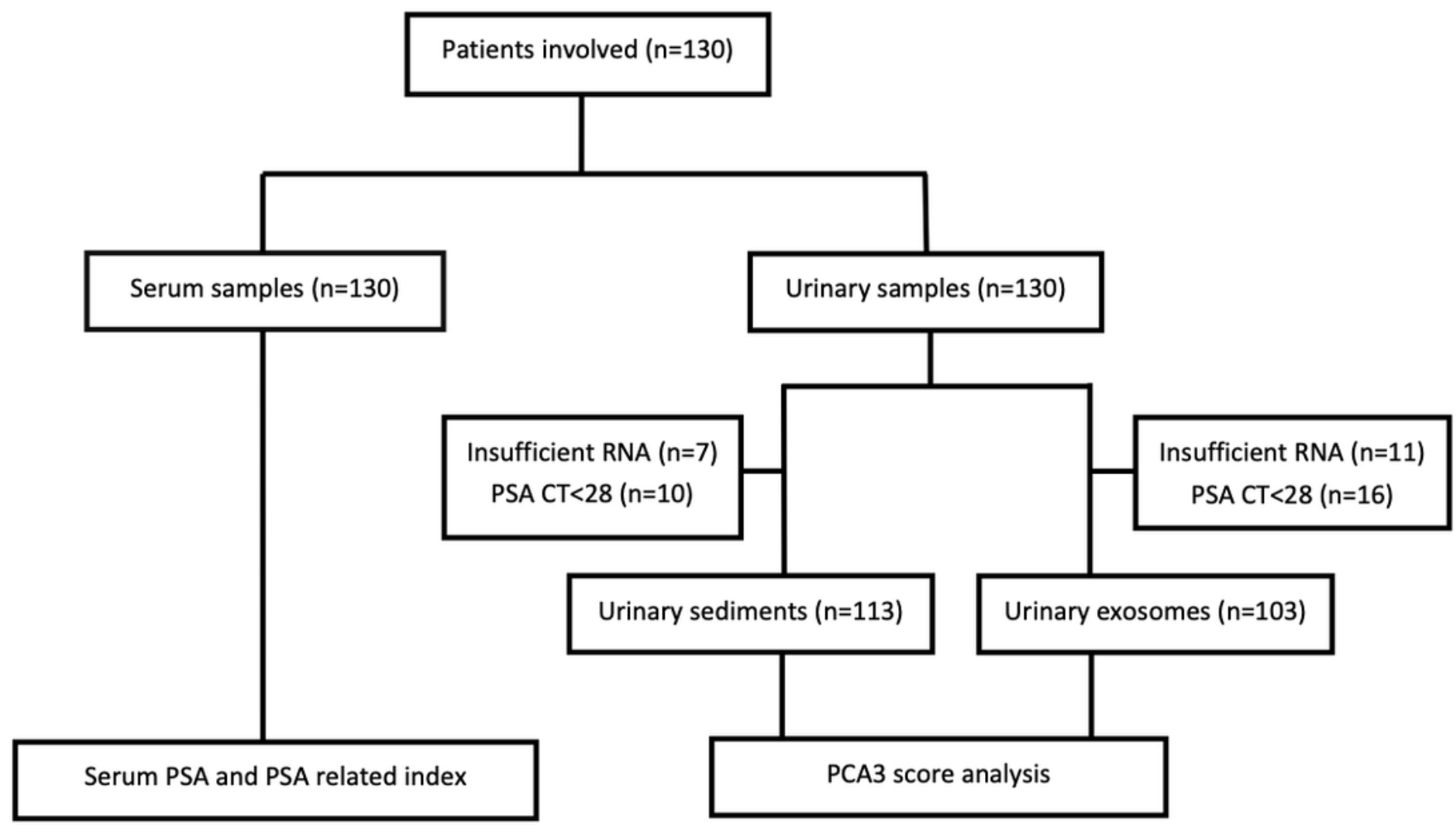

Figure 1

Flowchart of the study. $n=$ number of patients 

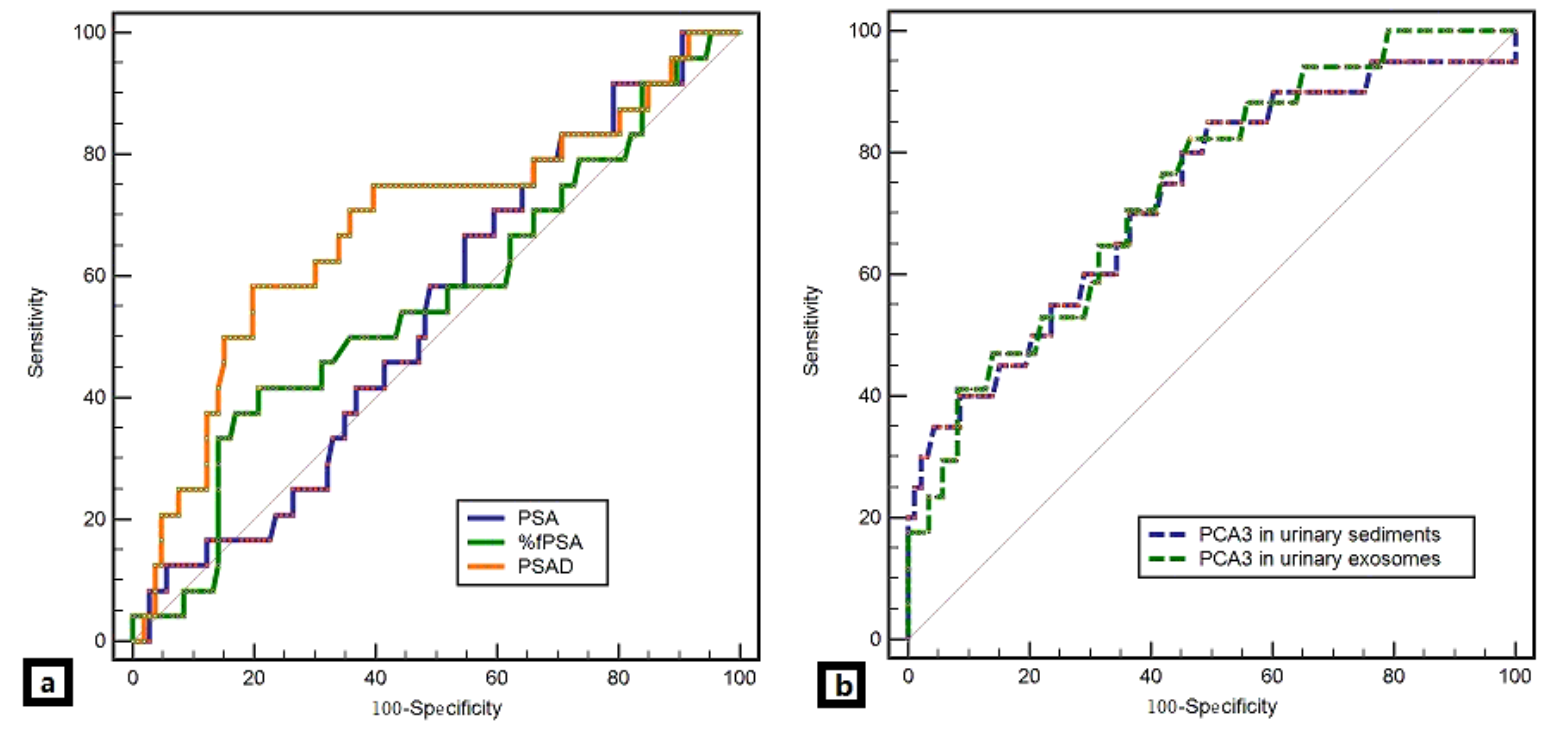

Figure 2

ROC curve analysis (a) ROC curve analysis of the PSA, \%fPSA and PSAD in patients with PSA levels of $4.0-10.0 \mathrm{ng} / \mathrm{ml}(\mathrm{n}=130)(\mathrm{b})$ ROC curve analysis of the PCA3 score in urinary sediments $(n=113)$ and urinary exosomes groups $(n=103)$. 